\title{
A molecular chaperone for mitochondrial complex I assembly is mutated in a progressive encephalopathy
}

\author{
Isla Ogilvie, ${ }^{1}$ Nancy G. Kennaway, ${ }^{2}$ and Eric A. Shoubridge ${ }^{1,3}$ \\ ${ }^{1}$ Montreal Neurological Institute, McGill University, Montreal, Quebec, Canada. ${ }^{2}$ Department of Molecular and Medical Genetics, \\ Oregon Health \& Science University, Portland, Oregon, USA. ${ }^{3}$ Department of Human Genetics, McGill University, Montreal, Quebec, Canada.
}

\begin{abstract}
NADH:ubiquinone oxidoreductase (complex I) deficiency is a common cause of mitochondrial oxidative phosphorylation disease. It is associated with a wide range of clinical phenotypes in infants, including Leigh syndrome, cardiomyopathy, and encephalomyopathy. In at least half of patients, enzyme deficiency results from a failure to assemble the holoenzyme complex; however, the molecular chaperones required for assembly of the mammalian enzyme remain unknown. Using whole genome subtraction of yeasts with and without a complex I to generate candidate assembly factors, we identified a paralogue $(B 17.2 L)$ of the B17.2 structural subunit. We found a null mutation in $B 17.2 \mathrm{~L}$ in a patient with a progressive encephalopathy and showed that the associated complex I assembly defect could be completely rescued by retroviral expression of B17.2L in patient fibroblasts. An anti-B17.2L antibody did not associate with the holoenzyme complex but specifically recognized an 830-kDa subassembly in several patients with complex I assembly defects and coimmunoprecipitated a subset of complex I structural subunits from normal human heart mitochondria. These results demonstrate that B17.2L is a bona fide molecular chaperone that is essential for the assembly of complex I and for the normal function of the nervous system.
\end{abstract}

\section{Introduction}

NADH:ubiquinone oxidoreductase (complex I) catalyzes the first step in the mitochondrial respiratory chain, in which transfer of electrons from $\mathrm{NADH}$ to ubiquinone (coenzyme $\mathrm{Q}$ ) is accompanied by the translocation of protons across the inner mitochondrial membrane. This contributes to the proton electrochemical gradient used to synthesize ATP by complex V, the ATP synthetase. In prokaryotes, complex I is composed of a basic core of 14 structural subunits thought to have evolved from the fusion of separate modules for electron transfer and proton transport (1). Over the course of evolution, a number of so-called supernumerary subunits have been added to the complex, which now comprises 37-40 subunits in aerobic fungi and at least 46 in mammals, 7 of which are encoded in mtDNA (2-4). The complex forms an L-shaped structure, with a hydrophobic arm containing the mtDNA-encoded subunits that is embedded in the inner mitochondrial membrane and a hydrophilic peripheral arm containing the NADH binding site and redox active centers that extends into the mitochondrial matrix $(5,6)$.

Disease-causing mutations have now been described in 9 of the nuclear-encoded structural subunits of complex I (NDUFS1, NDUFS2, NDUFS3, NDUFS4, NDUFS7, NDUFS8, NDUFV1, NDUFS6, and NDUFV2) (7-15). These are associated with a variety of autosomal recessive, early onset, fatal clinical phenotypes including Leigh syndrome, cardiomyopathy, and encephalomyopathy. A survey of nearly all of the genes coding for structural subunits in 1

Nonstandard abbreviations used: BN-PAGE, blue native PAGE; complex I, NADH: ubiquinone oxidoreductase; DDM, dodecylmaltoside; EMG, electromyogram; NCV, nerve conduction velocity.

Conflict of interest: The authors have declared that no conflict of interest exists.

Citation for this article: J. Clin. Invest. 115:2784-2792 (2005).

doi:10.1172/JCI26020. cohort of autosomal recessive complex I patients failed to identify mutations in about $60 \%$ of the cases (16), suggesting that factors involved with assembly or maintenance of complex I are an important cause of disease; however, little is known about the regulation of these processes in mammals.

The model organism most extensively used for the study of complex I biogenesis is the aerobic fungus Neurospora crassa. The enzyme in $N$. crassa assembles the matrix and membrane arms as independent modules, which are then joined together in a stepwise fashion to form the holoenzyme (17). Mutations in structural subunits that prevent assembly of one or the other arms result in the accumulation of the unaffected arm. The membrane arm is itself formed from the association of a small and a large subcomplex, and 2 assembly proteins, CIA30 and CIA84, play a role in this process by transiently associating with the large membrane arm subcomplex (18). CIA30 has a mammalian homologue, but its function in mammalian cells has not yet been demonstrated, and mutations in this gene have not been identified in patients with complex I assembly defects (19).

It has been argued that assembly of the human complex I occurs by a mechanism similar to that in Neurospora (20); however, the presence of independent subcomplexes of the membrane and peripheral arms has not been convincingly demonstrated, while subcomplexes containing components of both arms have been identified in the skeletal muscle of some patients with complex I assembly defects (21). Additionally, mutations in structural subunits of the membrane arm (22) that prevent assembly of the holoenzyme do so without the apparent accumulation of subcomplexes.

In this study, we sought to identify complex I assembly factors using a bioinformatics approach. We show that $B 17.2 L$, a paralogue of a gene encoding a small structural subunit in the matrix arm, serves as a complex I assembly factor and, when mutated, causes an early onset, progressive encephalopathy. 


\begin{tabular}{|c|c|c|c|c|c|c|c|c|c|c|c|c|c|c|}
\hline A & 1 & 10 & 20 & 30 & 40 & 50 & 60 & 70 & 80 & 90 & 100 & 110 & 120 & 130 \\
\hline $\begin{array}{r}\text { B17.2 } \\
\text { B17.2L } \\
\text { Consensus }\end{array}$ & \multicolumn{14}{|c|}{ 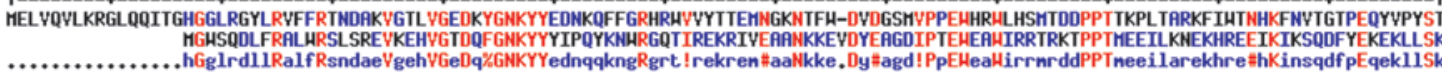 } \\
\hline & 131 & 140 & 150 & 160 & 170 & 180 & & & & & & & & \\
\hline $\begin{array}{r}\text { B17.2 } \\
\text { B17.2L } \\
\text { Consensus }\end{array}$ & \multicolumn{14}{|c|}{ 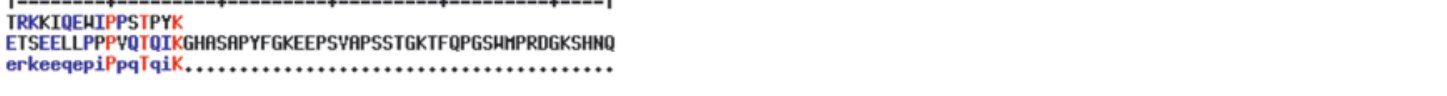 } \\
\hline B & 1 & 10 & 20 & 30 & 40 & 50 & 60 & 70 & 80 & 90 & 100 & 110 & 120 & 130 \\
\hline $\begin{array}{r}\text { Human } \\
\text { Mouse } \\
\text { Xenopus } \\
\text { Zebra fish } \\
\text { Yarrowia } \\
\text { Consensus }\end{array}$ & \multicolumn{14}{|c|}{ 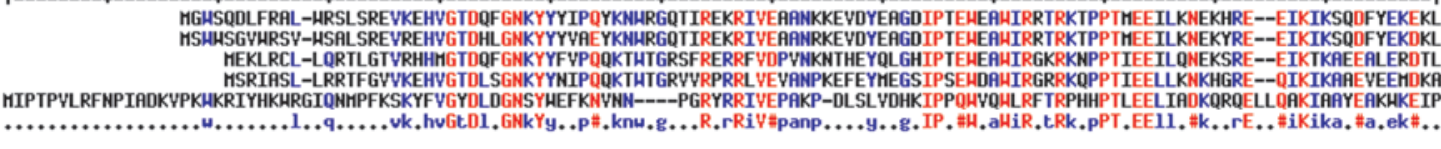 } \\
\hline & 131 & 140 & 150 & 160 & 170 & 180 & 190 & 200 & 210 & 220 & 230 & 2484 & & \\
\hline $\begin{array}{l}\text { Human } \\
\text { Mouse } \\
\text { Xenopus } \\
\text { Zebra fish } \\
\text { Yarrowia } \\
\text { Consensus }\end{array}$ & \multicolumn{14}{|c|}{ 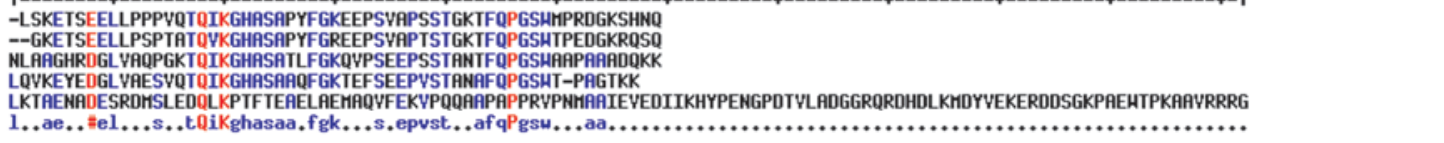 } \\
\hline
\end{tabular}

Figure 1

Alignment of predicted protein sequences for human B17.2 and B17.2L (A) and multiple alignment of B17.2L (B) showing conservation of the protein from yeast to human.

\section{Results}

Identification of candidate complex I assembly genes. The fermentative yeast Saccharomyces cerevisiae is the model organism most extensively used to study mitochondrial oxidative phosphorylation; however, it lacks a complex I, and this has hampered research on complex I biogenesis. Recently, the genomes of several other yeast species have been sequenced in their entirety, and 2 of these, Yarrowia lipolytica and Debaryomyces hansenii, are aerobic yeasts that express a complex I. We reasoned that it should be possible to find complex I assembly genes by using whole genome subtraction to identify mitochondrial proteins common to aerobic yeasts but missing in fermentative yeasts. To test this idea, we first evaluated all predicted proteins in gene families common to Y. lipolytica and D. hansenii but absent in S. cerevisiae, Kluyveromyces lactis, and Candida glabrata (1080 sequences in 472 families, classified on the Genolevures website) for mitochondrial targeting using the MITOPRED program. This produced 141 families in which 1 or more members were predicted to be mitochondrial. We then used PSI-Blast searches to identify mammalian homologues of these proteins. These were retested using MITOPRED and MitoProt II for mitochondrial targeting. This strategy would be expected to pick up the majority of nuclear-encoded complex I structural sub- units, and indeed, we were able to identify 16 of the 27 predicted nuclear encoded subunits in Y. lipolytica with human homologues (2). In addition, we identified the orthologues of the only known complex I assembly factors, CIA30 and CIA84, which have been characterized in the fungus $N$. crassa. After exclusion of these complex I structural subunits and assembly factors, we were left with 36 gene families. Manual curation removed a further 22 families where the function of the protein was already well characterized or the targeting prediction was obviously wrong.

Among the genes revealed by this search, 1, which we called $B 17.2 L$ (for B17.2-Like), seemed like a particularly good candidate as a complex I assembly factor. B17.2L is a paralogue of B17.2, a gene that codes for a small structural subunit in the matrix arm of complex I. While not present in the nuo operon, which contains the core 14 structural subunits of complex I in prokaryotes, the B17.2 structural subunit is present in the genome of $\alpha$-proteobacteria (COG 3761), the direct ancestors of mitochondria. It is absent in other bacteria, including Escherichia coli.

Alignment of the B17.2 and B17.2L predicted proteins showed that they are $17 \%$ identical and $31 \%$ similar, with no large blocks of sequence conservation (Figure 1A). The B17.2L gene appears to
A

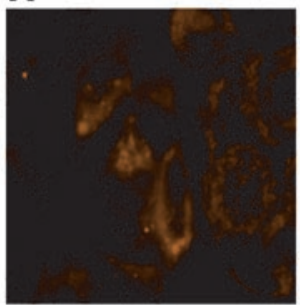

MitoTracker

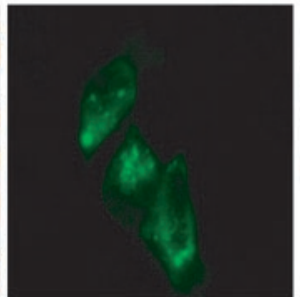

B17.2L-GFP

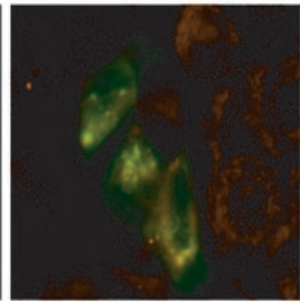

Merge
B

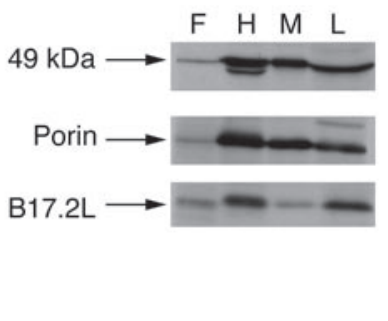

Figure 2

Mitochondrial localization and tissue-specific expression of B17.2L. (A) HEK293 cells were transfected with a vector expressing a B17.2L-GFP fusion protein, stained with MitoTracker red (a mitochondrial marker), and viewed on a fluorescence microscope. The overlay of the 2 images shows complete superposition of the 2 markers, demonstrating the mitochondrial localization of B17.2L. (B) Immunoblot analysis of B17.2L in human tissues. An anti$\mathrm{B} 17.2 \mathrm{~L}$ antibody was used to measure the steady-state levels of $\mathrm{B} 17.2 \mathrm{~L}$ in normal human fibroblasts $(\mathrm{F})$, heart $(\mathrm{H})$, skeletal muscle $(\mathrm{M})$, and liver $(\mathrm{L})$ by immunoblot analysis. Porin, an outer mitochondrial membrane protein, and the 49-kDa structural subunit of complex I are shown for comparison. 

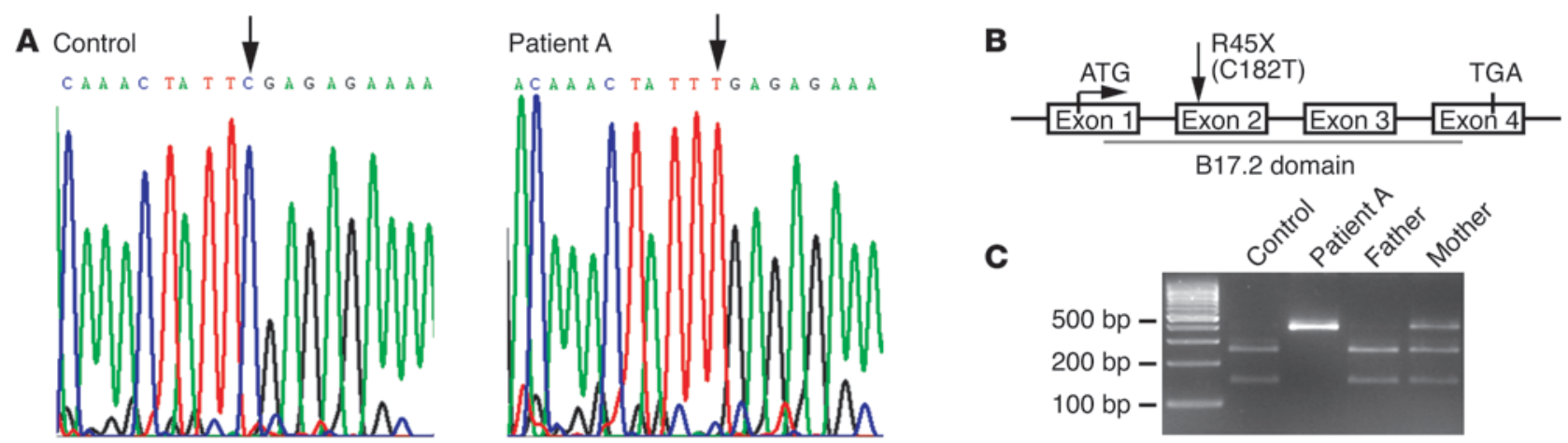

\section{Figure 3}

Analysis of the B17.2L gene in a patient with a complex I assembly defect. (A) DNA sequence analysis of the B17.2L cDNA showing C182T mutation in the patient. (B) Predicted B17.2L gene structure showing the position of the mutation and the predicted nonsense codon in exon 2 of the gene. The gray line below the gene representation identifies the position of the B17.2 domain. (C) Restriction enzyme-based analysis of the exon 2 mutation in genomic DNA from the patient and the parents. The mutation in the patient destroyed a site for the restriction enzyme Taq1.

be an ancient duplication of the gene for the structural subunit, as it is present in all eukaryotes whose genomes have been sequenced, except those fungi that lack complex I, but it is absent in prokaryotes (4). A multiple sequence alignment of B17.2L in the vertebrates and Y. lipolytica showed a relatively high degree of sequence homology in the $\mathrm{N}$ terminal part of the protein and the loss of a long $\mathrm{C}$ terminal tail in the vertebrates (Figure 1B). Overall the yeast protein is $20 \%$ identical and $34 \%$ similar to the human protein.

B17.2L maps to human chromosome $5 \mathrm{q}$, and there is a pseudogene on human chromosome 2 . It is likely to be a soluble protein, as it is not predicted to have a transmembrane domain (TMpred or TMHMM).

Mitochondrial targeting and expression of B17.2L in human tissues. The $\mathrm{B} 17.2 \mathrm{~L}$ protein product is predicted to be mitochondrially targeted by MITOPRED (100\%) and MitoProt II (97\%). To test this, we transiently expressed a B17.2L-GFP fusion protein in HEK293 cells and compared its distribution with that of MitoTracker red, a mitochondrial marker. Superposition of the 2 images showed complete overlap of the red and green fluorescent signals (Figure 2A), confirming the mitochondrial targeting of B17.2L. Immunoblot analysis showed that B17.2L is ubiquitously expressed, although there is some variation in the steady-state level of the protein among tissues when compared to other mitochondrial markers (Figure 2B). In particular, there is less B17.2L

\section{Figure 4}

Rescue of the complex lassembly defect in patient fibroblasts. (A) BN-PAGE analysis of the complex I assembly defect in the patient (lane A) shows a severe reduction in the activity of the enzyme as measured by an in-gel activity assay (upper panel) and the amount of the holoenzyme as measured by immunoblot analysis of the BN gel using an anti-ND1 antibody (middle panel) compared with control (lane C). Transduction of the patient cells with a retroviral vector expressing the wild-type $B 17.2 L$ cDNA (lanes A+B17.2L) completely rescued the biochemical defect. The supercomplex (complex I and III) is labeled $\alpha$. The lower panel shows an immunoblot of the loading control (70-kDa subunit of complex II). (B) Immunoblot analysis of fibroblasts with an anti-B17.2L antibody showing the complete absence of full-length B17.2L in patient fibroblasts (lane A) compared with control (lane C) and the accumulation of near control levels of the protein after transduction with a retroviral vector expressing the wild-type B17.2L cDNA (lane A+B17.2L). in skeletal muscle relative to either porin or the $49-\mathrm{kDa}$ complex I subunit as compared with heart, liver, or fibroblasts.

Mutation in B17.2L in a patient with a deficiency in complex I assembly and a progressive encephalopathy. We initially sequenced the cDNA for $\mathrm{B} 17.2 \mathrm{~L}$ in 8 patients in whom we had previously characterized complex I assembly defects. We found an apparently homozygous C182T mutation in exon 2 of $B 17.2 \mathrm{~L}$, predicting a premature stop at amino acid $45(\mathrm{R} 45 \mathrm{X})$ in the protein in a patient with a progressive encephalopathy (Figure 3, A and B). Sequencing of exon 2 in genomic DNA showed the same apparently homozygous mutation, which was confirmed by RFLP analysis of a PCR product containing the mutated

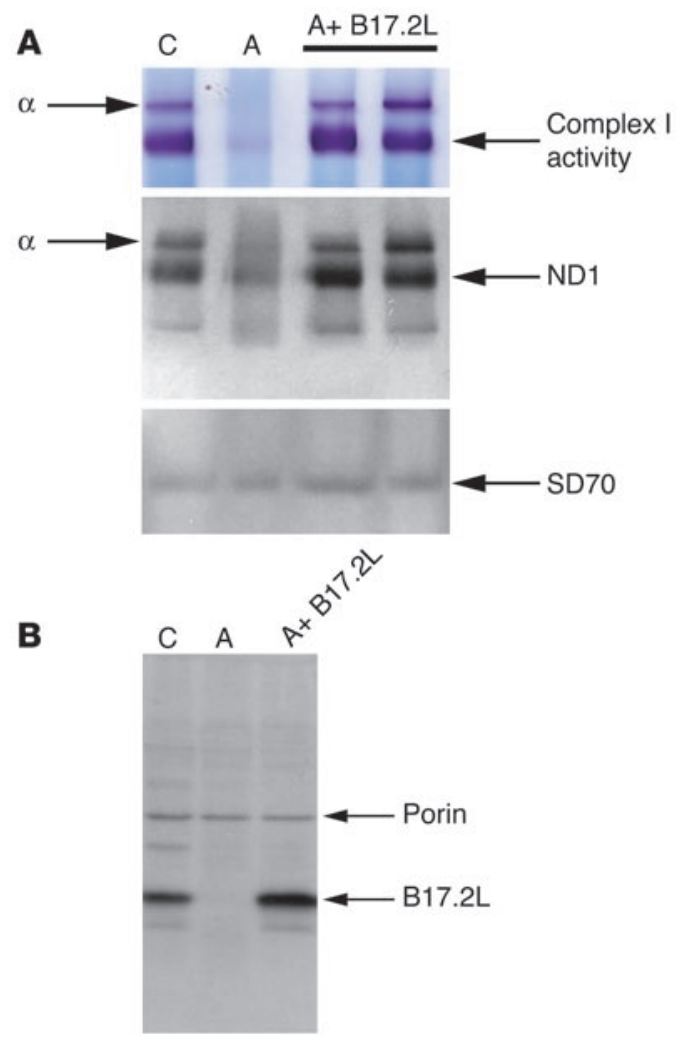



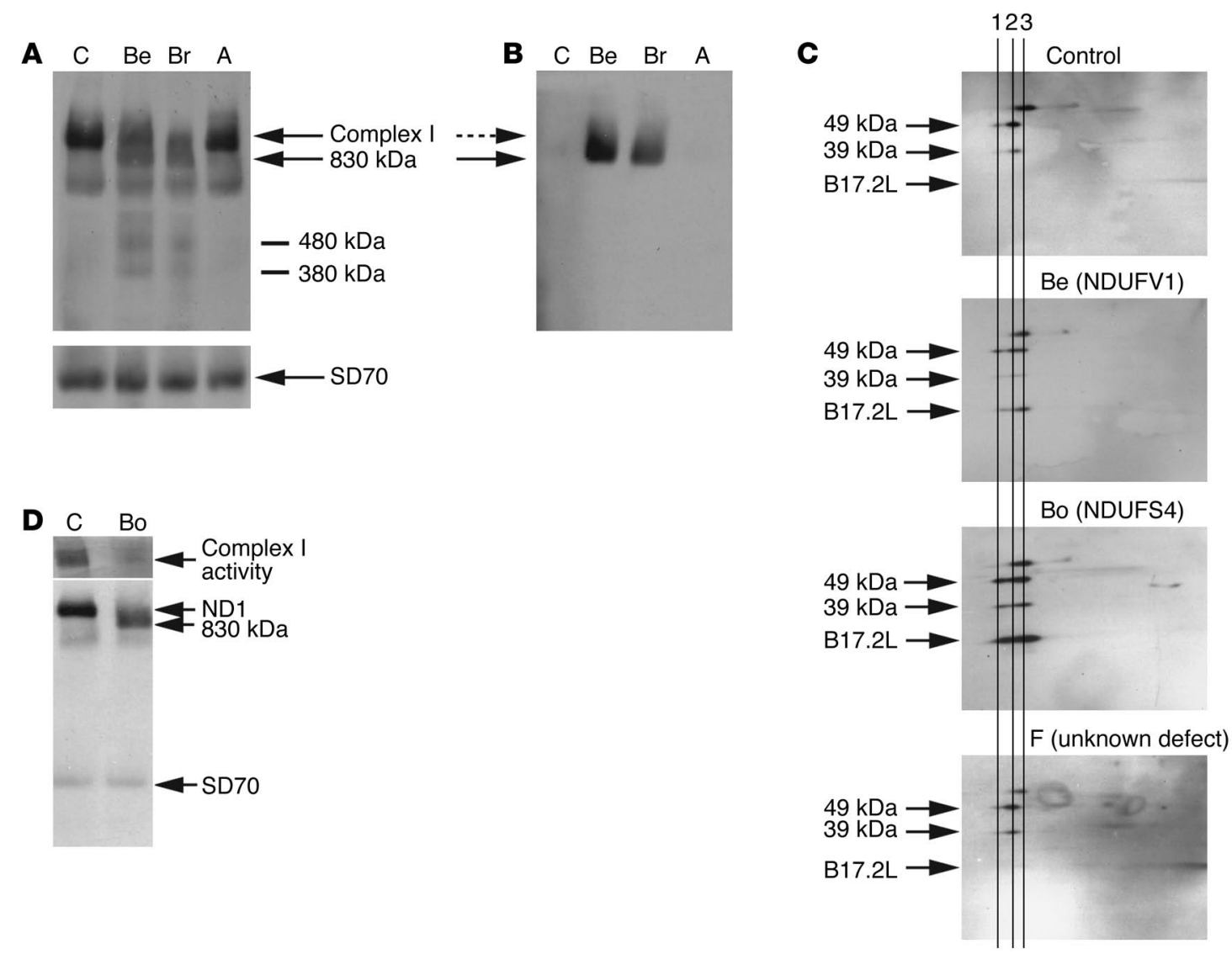

\section{Figure 5}

Analysis of expression of B17.2L by BN-PAGE immunoblot. (A and B) Mitochondria from control (lane C) and patient (lanes Be, Br, and A) muscle were subjected to first-dimension BN-PAGE analysis. The gel was immunoblotted and reacted with antibodies directed against ND1 (A) or B17.2L (B). The lower panel in A is an immunoblot of the loading control (70-kDa subunit of complex II). (C) BN-PAGE analysis of complex I assembly in patient fibroblasts. Mitoplasts were isolated from control and patient fibroblasts and subjected to second-dimension BN-PAGE analysis. A cocktail of antibodies directed against the indicated proteins was used to probe the second-dimension immunoblots. The lines labeled 1-3 on the gel indicate the position of the complex I-III supercomplex, fully assembled complex I, and the 830-kDa-complex III subcomplex, respectively. The spot that runs above the 49-kDa subunit and intersects with line 3 is a nonspecific protein recognized by the anti-49-kDa antibody. $\mathrm{B}$ 17.2L runs at its approximate monomeric $\mathrm{MW}$ in control and patient $\mathrm{F}$ (smear at the extreme right of the gel) and with an $830-\mathrm{kDa}$ subcomplex of complex I in the patients with the known structural subunit gene mutations (Be and Bo). (D) First-dimension BN-PAGE gel demonstrating the complete absence of fully assembled complex I in muscle mitochondria from patient Bo.

site (Figure 3C). A restriction enzyme-based analysis of a PCR product from genomic DNA encompassing exon 2 from both parents showed that the mother was heterozygous for the mutation; however, the father appeared homozygous for the wild-type sequence. This suggested to us that the patient may have inherited 2 alleles from her mother (isodisomy) or that the patient was in fact hemizygous for the mutant allele, having inherited a deleted allele from her father. Using microsatellite markers covering chromosome 5, we found that $16 / 18$ markers were heterozygous in the patient (data not shown), making maternal isodisomy unlikely and suggesting that the father transmitted a deleted allele to his daughter and that both are hemizygous for B17.2L. Sequencing of a further 20 patients with complex I deficiency failed to identify additional mutations in B17.2L.

Functional complementation of the complex I defect in patient fibroblasts. To test whether the R45X mutation was in fact the disease-causing mutation, we transduced patient fibroblasts with a retroviral vector expressing the wild-type B17.2L cDNA and looked for rescue of the complex I assembly defect by blue native PAGE (BN-PAGE) and res- toration of complex I catalytic activity using an in-gel assay. In 2 independent experiments, both the amount of fully assembled complex I and its activity were restored to control levels in cells transduced with the vector expressing B17.2L (Figure 4A). The amount and activity of the complex I-III supercomplex were also restored to control levels.

We raised an antibody against a $\mathrm{C}$ terminal peptide in $\mathrm{B} 17.2 \mathrm{~L}$ and used immmunoblot analysis to examine the effect of the mutation on the protein and its expression in cells transduced with B17.2L. There was no detectable immunoreactivity for the full-length protein in the patient cells, but expression of the wildtype cDNA from the retroviral vector restored it to about twice the level observed in controls (Figure 4B).

$B 17.2 \mathrm{~L}$ is an assembly protein that associates with subassembly of complex $I$. To investigate the function of B17.2L, we used BN-PAGE to analyze the composition of complex I subassemblies in muscle mitochondria from 2 siblings with a complex I assembly defect in whom we had previously identified mutations in NDUFV1, which codes for the 51-kDa complex I structural subunit (unpublished results). These 
A

C $\mathrm{Be}$ Br Bo Ly $\mathrm{O}$ A $\mathrm{F}$ M

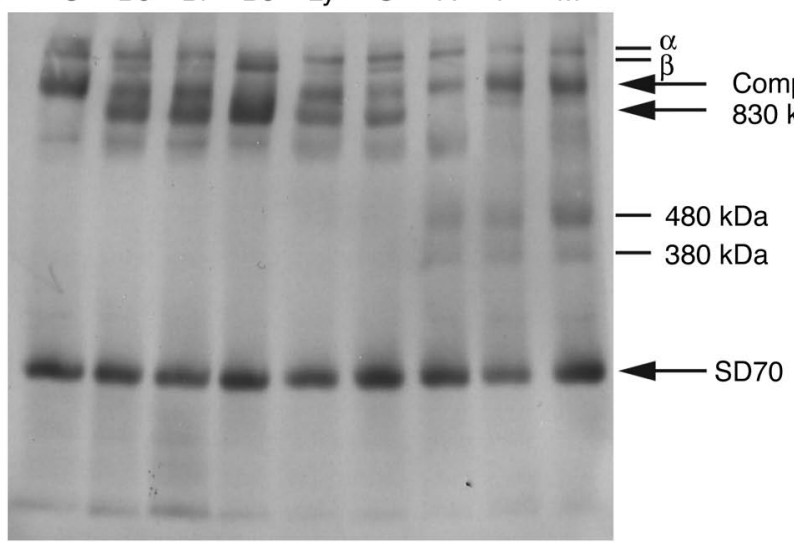

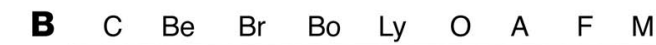

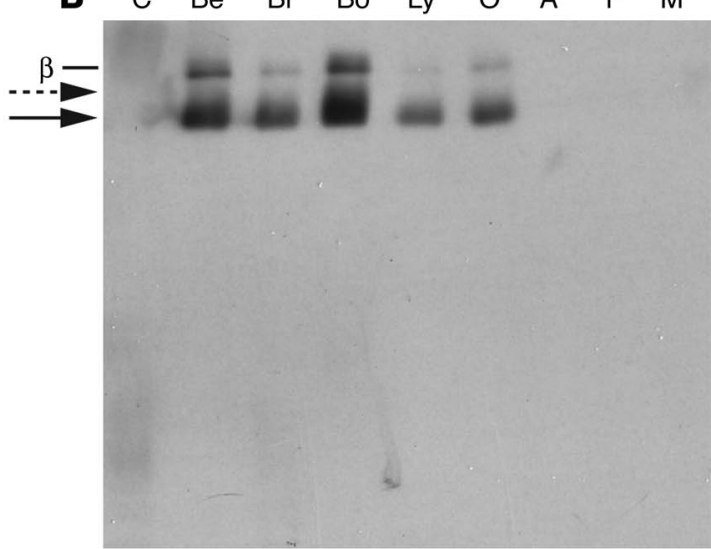

Figure 6

BN-PAGE analysis of complex I assembly in patient fibroblasts. Mitoplasts were isolated from control and patient fibroblasts and subjected to first-dimension BN-PAGE analysis. The gels were immunoblotted and reacted with antibodies against ND1 (A) and B17.2L (B). The complex I-III supercomplex is indicated as $\alpha$, and the supercomplex between the $830-\mathrm{kDa}$ complex I subcomplex and complex III is indicated as $\beta$.

patients fail to assemble normal amounts of the holoenzyme, and accumulate complex I subassemblies (Figure 5A). The first dimension $\mathrm{BN}$ gel showed anti-B17.2L immunoreactivity in a subcomplex of about $830 \mathrm{kDa}$ in both patients (Figure 5B). No immunoreactivity was detectable in the control, or, as expected, in the B17.2L patient who is null for the $\mathrm{B} 17.2 \mathrm{~L}$ protein (Figure $5 \mathrm{~B}$ ). This result suggests that B17.2L associates with a specific subassembly of complex I but not with the mature holoenzyme. To ensure that this represented real immunoreactivity for B17.2L, we analyzed samples from 1 of the above patients and from 2 other patients with complex I assembly defects on a second-dimension denaturing gel (Figure 5C). One of the additional patients (Bo) has a mutation in NDUFS4 (18-kDa subunit, our unpublished results) and accumulates the $830-\mathrm{kDa}$ subcomplex (and a supercomplex that contains this subcomplex and complex III) but does not assemble any of the mature holoenzyme (Figure 5D). The molecular defect in the other patient $(\mathrm{F})$ remains unknown. Immunoreactivity for B17.2L is clearly present in the $830-\mathrm{kDa}$ subcomplex from the 2 patients with the known structural subunit defects (Figure 5C). It appears as a short smear at its approximate monomeric molecular weight in the control and in patient $\mathrm{F}$.

To test the generality of these results we examined fibroblast cell lines from 2 other patients who accumulate the 830-kDa subcomplex and 2 additional patients with assembly defects who do not accumulate this subcomplex at detectable levels (Figure 6A). This analysis clearly showed B17.2L immunoreactivity associated with the $830-\mathrm{kDa}$ complex (patients Be, Br, Bo, Ly, and O) but not in lower molecular weight (380 and $480 \mathrm{kDa}$ ) subassemblies (visible in patients $\mathrm{F}$ and M) (Figure 6B). B17.2L immunoreactivity was also observed in a higher molecular-weight complex of greater then $1 \mathrm{MDa}$ (labeled $\beta$ in Figure 6A). This likely represents a supercomplex (23), in which the 830-kDa subassembly associates with complex III.

Immunoprecipitation of a complex I subassembly with anti-B17.2L. To investigate which components of complex I associate with B17.2L in normal cells, we tested whether we could coimmunoprecipitate complex I subunits from normal human heart mitochondria using the anti-B17.2L antibody (Figure 7A). We tested for the presence of 9 different complex I subunits using specific antibodies and for subunits of complexes II and IV to control for nonspecific interac- tions. Quantitative analysis (Figure 7B) showed that the immunoprecipitate was relatively enriched for ND1 and the 49-, 75-, 18-, 51-, and 30-kDa subunits whereas GRIM19 was recovered at levels only slightly greater than COX-1, and 2 complex I subunits, B14 and the $39-\mathrm{kDa}$ subunit, were not detectable in the immunoprecipitate.

\section{Discussion}

Several lines of evidence implicate B17.2L as a molecular chaperone that is essential for the efficient assembly of complex I. First, a complex I assembly defect in the patient with a progressive encephalopathy described here is associated with a premature stop mutation in $B 17.2 \mathrm{~L}$ that results in the absence of full-length B17.2L protein. When the wild-type $B 17.2 \mathrm{~L}$ cDNA is expressed in patient fibroblasts, B17.2L protein accumulates, and the biochemical defect is completely rescued. Second, B17.2L specifically associates with an $830-\mathrm{kDa}$ subassembly of complex I in several patients with assembly defects, but it is not present in the mature holoenzyme. Third, an anti-B17.2L antibody specifically coimmunoprecipitates a subset of complex I structural subunits from mitochondria isolated from normal human heart. Finally, B17.2L is present in all sequenced eukaryotes that contain a complex I but absent in those that do not.

Although the B17.2L protein does not share a high degree of sequence identity with the B17.2 structural subunit, the genes coding for both are clearly paralogues. This study and a recent phylogenetic analysis of complex I (4) indicated that $B 17.2 \mathrm{~L}$ arose as an ancient duplication of the gene coding for the structural subunit in the eukaryotic lineage. Interestingly, B17.2L was also very recently identified as a mitochondrial protein of unknown function in a screen for transcriptional targets of c-myc (24). Knockdown of the protein, which the authors called mimitin (for myc-induced mitochondrial protein), by RNAi was shown to inhibit the growth of some tumor lines. In light of the data we present here, it seems likely that this effect was mediated by a decrease in the content and activity of complex I.

We do not know exactly when B17.2L is recruited to facilitate the assembly of the nascent complex I; however, the observation that B17.2L specifically associates with a large subassembly of $830 \mathrm{kDa}$ indicates that it is required until the late stages of assembly, as the 
A

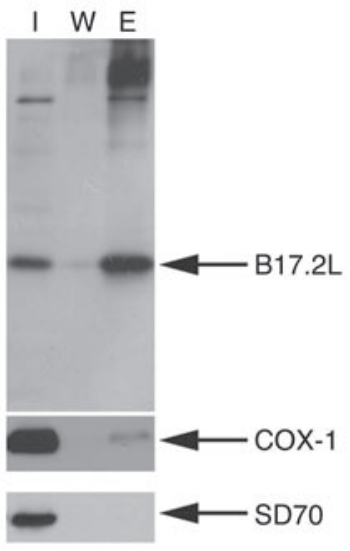

B

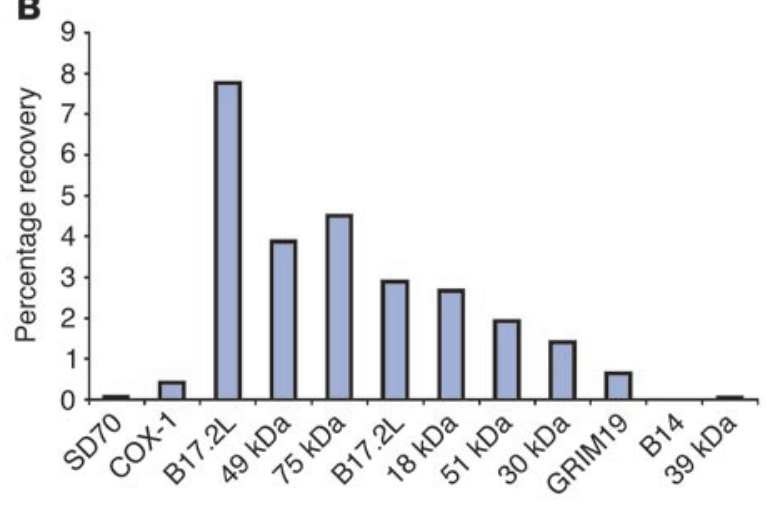

I W E

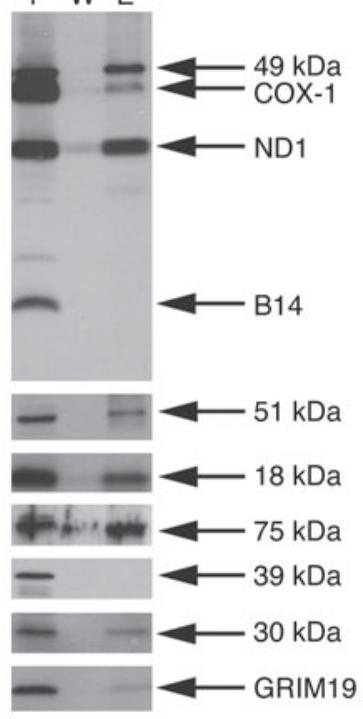

\section{Figure 7}

Immunoprecipitation of B17.2L and a subset of complex I subunits from normal human heart mitochondria. Mitochondria were isolated from normal human heart and immunoprecipitated with an immobilized antiB17.2L antibody. (A) The immunoprecipitate was probed for the presence of $\mathrm{B} 17.2 \mathrm{~L}$ and the specific complex I subunits indicated to the right of the gels. COX-1, a subunit of complex IV, and SD70, a subunit of complex II, were used to control for nonspecific interactions. Lane I shows a sample of the mitochondrial protein applied to the immobilized antibody. Lane $\mathrm{E}$ represents half of the sample obtained following elution of the protein that specifically bound to the immobilized antibody. Lane W shows a sample of the final wash in the same volume as the eluate. (B) Quantitative analysis of the proteins immunoprecipitated by the anti-B17.2L antibody as obtained by densitometric analysis of the immunoblots.

complex is too large to represent either of the independent arms. Further, we clearly detected subunits from both arms of the enzyme in the $830-\mathrm{kDa}$ subcomplex by immunoblot analysis and in the subcomplex immunoprecipitated with the anti-B17.2L antibody. Interestingly, 2 of the complex I subunits that did not coimmunoprecipate with B17.2L (B14 and the 39-kDa subunit) have been placed in one model of complex I (4) near the junction of the matrix and membrane arms. Thus we could speculate that B17.2L is somehow involved in stabilizing a large complex I subassembly containing elements of both arms of the enzyme, in which this junction has not fully matured. Although our previous studies (21) established that both the B14 and B39 subunits are present in the $830-\mathrm{kDa}$ complex, it is possible that they are added at this stage in wildtype cells and that the subcomplex we identified in patient cells represents an intermediate that remains stalled after their addition because key structural subunits are missing in the patients.

The complete absence of full-length B17.2L does not entirely prevent assembly of mature complex I as the BN-PAGE in gel and spectrophotometric enzyme assays showed the presence of residual enzyme activity in the patient cells and tissues. This is reminiscent of the situation in patients with loss of function SURF1 mutations (29), the vast majority of whom have no immunodetectable Surf1 protein but can nevertheless make $15-20 \%$ of holocomplex IV $(30,31)$. Thus these molecular chaperones may serve as facilitators to bring some of the components of the enzyme together, perhaps by stabilizing subassemblies of the complex as argued above, thereby increasing the efficiency of the process. In the case of B17.2L, there seems to be some tissue specificity in the severity of the biochemical defect. The deficiency in the patient's skeletal muscle was rather modest (about $40 \%$ of control activity) while the defect in fibroblasts was more striking (less than $20 \%$ of control activity). Paradoxically, the expression of $\mathrm{B} 17.2 \mathrm{~L}$ was lowest in muscle compared with the other tissues we examined, including fibroblasts (Figure 2B). Again, there are some parallels with deficiencies in complex IV due to assembly gene mutations that variously affect the heart, liver, brain, or skeletal muscle depending upon the nature of the gene defect (32-34). The basis for these tissue-specific effects in ubiquitously expressed housekeeping genes remains unknown, but it seems likely to involve differential regulation of assembly to meet tissue-specific metabolic demands.

The most common clinical phenotype associated with pediatric complex I cases is Leigh syndrome, or subacute necrotizing encephalopathy (35). This neurodegenerative disorder is associated with bilaterally symmetrical spongiform lesions, especially in the thalamus and brainstem (36). The clinical presentation of the B17.2L patient does not resemble that of Leigh syndrome patients, 
A

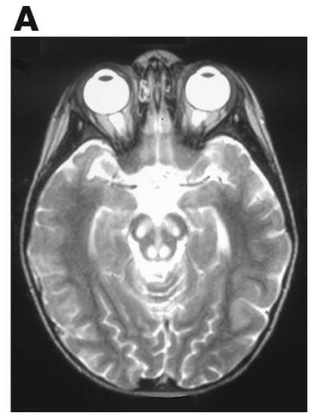

C

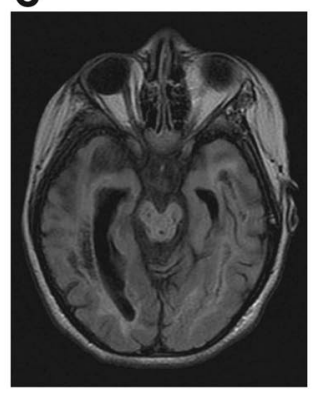

B

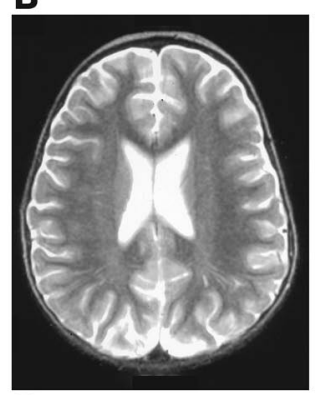

D

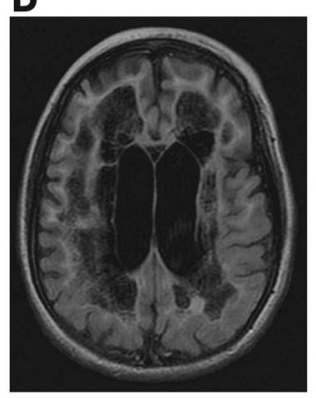

nor is it typical of most other patients with mitochondrial disease (though it must be said that the clinical spectrum of mitochondrial disease is extraordinarily broad). In fact, the patient shares most of the characteristic diagnostic criteria for a disease entity called leukoencephalopathy with vanishing white matter (37), which is caused by mutations in any of 5 different cytosolic translation factors (38). The fact that we have not identified other patients with B17.2L mutations may reflect the fact that this clinical phenotype has not generally been associated with mitochondrial disease.

Patients with complex I assembly defects have been assigned to several different genetic complementation groups (ref. 15 and our unpublished results), so it is unlikely that we will find a common gene defect in these cases. The genome subtraction strategy we have used to find the B17.2L gene might be useful for finding other complex I assembly genes, but it does have its limitations. The 2 most obvious arise from inaccuracies in the in silico mitochondrial targeting prediction algorithms (about $80 \%$ accurate) and the fact that proteins that are part of large gene families with members in many yeast species would not survive the screen. It is interesting that $B 17.2 L$, the first characterized mammalian complex I assembly factor gene, is a paralogue of a structural subunit of the complex itself. None of the assembly factors that have been identified for complexes III, IV, or V have any obvious relationship with the structural subunits of these complexes. A recent analysis of the evolution of complex I (4) identified several paralogs of complex I structural genes, and these must now also be considered as candidates for complex I assembly factors.

\section{Methods}

Case report. The patient was a full-term female, born by cesarean section for breech presentation to a mother of Chinese and Portuguese heritage and a Jewish father from the Caucasus. She seemed healthy until 12-14 months of age when horizontal nystagmus and wide-based gait were noted; brain MRI was normal. At 3 years of age, she developed acute ataxia, lethargy, dehydration, and worsening nystagmus following an ear infection. She had right dysconjugate gaze, pale right optic disc, myopathic facies, decreased

\section{Figure 8}

MRI of the brain of patient A. Transverse T2 weighted images taken at 3 years, 4 months of age (A and $\mathbf{B}$ ). Transverse Fluid Attenuated Inversion Recovery (FLAIR) images taken at 10 years, 3 months of age (C and $\mathbf{D})$.

reflexes, and normal sensory examination. Blood lactate was normal although CSF lactate was slightly increased (4.2 mM; controls 1.4-3.9 mM). Electromyogram (EMG) and nerve conduction velocity (NCV) tests suggested a demyelination, possibly a polyradiculoneuropathy. Brain MRI (Figure 8, A and B) showed symmetric lesions in the mamillothalamic tracts, substantia nigra/medial lemniscus, medial longitudinal fasciculus, and spinothalamic tracts. Confluent areas of hyperintense signal were also seen in the corpus medullare of the cerebellum. After 3 days of steroid therapy for presumed acute disseminated encephalomyelitis, hypertrophic cardiomyopathy was detected and steroid therapy was stopped. She was then given i.v. immunoglobulins and propranolol. She improved clinically and the cardiomyopathy resolved. Pyruvate dehydrogenase deficiency was excluded by activity measurement in peripheral blood lymphocytes.

Skeletal muscle and sural nerve biopsies were performed at Oregon Health $\&$ Science University at 44 months of age. Muscle and nerve histochemistry and muscle EM were normal. In particular, there were no ragged red fibers or cytochrome $c$ oxidase-negative fibers. Measurement of respiratory chain activities in muscle mitochondria revealed moderate deficiency of complex I (patient, $105 \mathrm{nmol} / \mathrm{min} / \mathrm{mg}$ protein; controls, $276 \pm 84 \mathrm{nmol} / \mathrm{min} / \mathrm{mg}$ protein). Activities of complexes II, III, IV, and II+III, ATPase, and citrate synthase were all normal. The amount and activity of complex I were less than $20 \%$ of control levels in immortalized patient fibroblasts as assessed by BN-PAGE and measurement of in-gel activity (see Figure 4). She was treated with vitamin C, coenzyme Q10, and L-carnitine. Clinically, she had normal intelligence, normal strength, hypotonia, absent deep tendon reflexes, mild ataxia, and optic atrophy. At age 6, she experienced another episode of increased lactic acidosis and worsening ataxia. She remained normal intellectually.

At 8 years of age, increasing weakness, dysphagia, dysuria, sleep apnea, hypertension, and tachycardia developed, and she suffered respiratory failure associated with an intercurrent respiratory infection. Following these events, she required a tracheostomy for mechanical ventilation, and a gastrostomy for feeding. She remained comatose for several months. She was treated with a vitamin cocktail, coenzyme Q10, carnitine, fish oil, uridine, and creatine. Dichloroacetate was given for 3 months following the respiratory failure. From 8 years, 6 months, until her death at 13 years, 7 months, she was largely immobile, verbally noncommunicative, and had recurrent pneumonias and electrolyte imbalance. She also had muscle wasting, absent deep tendon reflexes, severe optic atrophy, severe osteoporosis, scoliosis, central precocious puberty, temperature instability, and a microcytic anemia. T4 and TSH (thyroid stimulating hormone) were elevated; blood lactate, liver function, and echocardiogram were normal. EMG and NCV tests revealed severe axonal sensorimotor polyneuropathy. Brain MRI (Figure 8, C and D) showed extensive myelomalacia, white matter encephalomalacia, cortical atrophy, and vermian atrophy. She remained ventilator- and gastrostomy-dependent but responsive to music therapy and able to vocalize to indicate discomfort or unhappiness.

Neuropathological examination of the cerebral hemispheres revealed striking spongiform changes throughout the white matter and deep gray matter with relative sparing of the cortex. There was some preservation of $\mathrm{U}$ fibers in the white matter. The corpus callosum was reduced to a very thin lamina, and there was marked atrophy of the striatum and globus pallidum. The thalamus and hypothalamus were very atrophic, but the hippocampal formations were relatively spared. In the midbrain, the aqueduct of Sylvius was enlarged, and there were multicavity lesions involving 
the periaqueductal region that extended into the tegmentum of the pons. There was marked atrophy of the pons and medulla. There was marked atrophy of the folia throughout the vermis of the cerebellum, and the white matter of the cerebellum was severely involved in the spongiform changes. Microscopic examination of the cerebral cortex, hippocampus, and cerebellum showed extensive and bizarre degeneration of white matter, which was replaced by a glial scar. No oligodendrocytes were present.

Informed consent was obtained from the parents of the patient, and protocols were approved by the Oregon Health \& Science Institutional Review Board and the Research Ethics Board of the Montreal Neurological Hospital and Institute.

Genome subtraction. Files containing the sequences of gene families found in both D. hansenii and Y. lipolytica, but not in C. glabrata or K. lactis, were downloaded from the website of the Genolevures consortium (http://cbi. labri.fr/Genolevures) (39). The web-based program MITOPRED (http:// bioinformatics.albany.edu/ mitopred/) (40) was then used to predict which of the gene sequences were mitochondrial. Finally PSI Blast (http:// helix.nih.gov/docs/gcg/psiblast.html) was used with a Blosum 45 matrix to find human homologues (41), and a second iteration of mitochondrial prediction was done on these homologues using MITOPRED and MitoProt II (http://ihg.gsf.de/ihg/mitoprot.html) (42).

Cell lines. Primary skin fibroblasts were grown from patient skin biopsy material. To immortalize the cell lines, they were transduced with retroviral vectors expressing the catalytic component of human telomerase and the type 16 human papilloma virus E7 gene (29). The fibroblasts were grown in high glucose DMEM containing $110 \mathrm{mg} / \mathrm{l}$ pyruvate and $10 \%$ fetal bovine serum.

Sequencing of cDNA and genomic DNA. RNA was isolated from patient and control fibroblasts using the RNeasy Mini Kit (QIAGEN). cDNAs were generated by RT-PCR on total RNA from fibroblasts using the OneStep RT-PCR kit (QIAGEN) and specifically designed primers for the amplification of B17.2L. Exon 2 of B17.2L was amplified from genomic DNA using specific primers. PCR products were sequenced automatically using the same primers.

B17.2L cDNA constructs. Retroviral vectors were created using the Gateway cloning system (Invitrogen Corp.). cDNA from B17.2L was amplified by OneStep RT-PCR (QIAGEN). Specific primers modified for cloning into Gateway vectors were used so that the PCR products could be cloned into a retroviral expression vector, pLXSH (43), modified for use with the Gateway system. The fidelity of the cDNA clones was confirmed by automated DNA sequencing.

Virus production and transduction of patient fibroblast lines. The cDNA containing retroviral vectors were transiently transfected into a Phoenix amphotropic cell line (a kind gift of G.P. Nolan, Stanford University, Stanford, California, USA) using an HBS/CaPO${ }_{4}$ method (http://www.stanford.edu/ group/nolan/retroviral_systems/retsys.html). Patient and control fibroblasts were infected 48 hours later by exposure to virus-containing medium with polybrene $(4 \mu \mathrm{g} / \mathrm{ml})$ as previously described (44). Cells expressing the cDNA were selected for in $100 \mathrm{U} / \mathrm{ml}$ hygromycin and then harvested for BN-PAGE or SDS-PAGE analysis.

Preparation of human heart, muscle, liver, and fibroblast mitochondria. Mitochondria were prepared from $1 \mathrm{~g}$ of frozen control human heart tissue, $100 \mathrm{mg}$ of frozen control human liver, or $5 \times 15-\mathrm{cm}$ cell culture plates of fibroblasts from patients and controls. The tissues or cells were homogenized (5\%) in $250 \mathrm{mM}$ sucrose, $10 \mathrm{mM}$ Tris- $\mathrm{HCl}$, and $1 \mathrm{mM}$ EDTA (pH 7.4), and Complete protease inhibitors (Roche Diagnostics Corp.) were added to the homogenization buffer at the recommended concentration. To prepare mitochondria from muscle, $100 \mathrm{mg}$ of frozen muscle tissue from patients or controls was homogenized in $150 \mathrm{mM} \mathrm{KCl}, 20 \mathrm{mM}$ Tris- $\mathrm{HCl}$, and $2 \mathrm{mM}$ $\operatorname{EDTA}(\mathrm{pH} 7.5$ ). All of the above homogenates were centrifuged twice for 10 minutes at $600 \mathrm{~g}$ to obtain a postnuclear supernatant. Mitochondria were pelleted by centrifugation for 20 minutes at $14,000 \mathrm{~g}$. Protein concentration was measured by the Micro BCA method (Pierce Biotechnology Inc.).

$B N-P A G E$ and immunoblot analysis. Mitoplasts were prepared from fibroblasts, as described previously (45), by treating the cells with $0.8 \mathrm{mg}$ digitonin/mg of protein. Mitoplasts or mitochondria were solubilized with $1 \%$ lauryl maltoside, and 20-30 $\mu$ g (fibroblasts) or 10-15 $\mu$ g (tissues) of solubilized protein was used for electrophoresis. BN-PAGE (46) was used to separate the samples in the first dimension on $6-15 \%$ polyacrylamide gradients. An in-gel activity assay for complex I was performed as described (47). For analysis of the second dimension, strips of the first-dimension gel were incubated for 45 minutes in $1 \%$ SDS and $1 \% \beta$-mercaptoethanol. These strips were then run on a $10 \%$ tricine/SDS-PAGE to separate the proteins in the second dimension (46). Subunits of complexes I, II, and IV were detected by Western blot analysis of the above gels using monoclonal and polyclonal antibodies. The following antibodies were purchased from Invitrogen Corp.: complex I, anti-B14 (NDUFA6) subunit, complex II, anti-SD70 (70 kDa) subunit, and complex IV, anti-COX-1. Anti-GRIM19 was purchased from MitoSciences. Other antibodies against complex I subunits were kindly provided as follows: J. Walker (MRC Dunn Human Nutrition Unit, Cambridge, United Kingdom), anti-75-kDa (NDUFS1) and anti-51-kDa (NDUFV1) subunits; B. Robinson (Hospital for Sick Children, Toronto, Ontario, Canada), anti-49-kDa (NDUFS2) subunit; R. Capaldi (University of Oregon, Eugene, Oregon, USA), anti-39-kDa (NDUFA9) subunit; A. Lombes (INSERM U582, Université Pierre et Marie Curie, Paris, France), anti-ND1; and V. Petruzzella (University of Bari, Bari, Italy), anti-18-kDa (NDUFS4) subunit.

SDS-PAGE analysis. Whole cell extracts were prepared from fibroblasts by solubilization for 30 minutes at $4{ }^{\circ} \mathrm{C}$ with $1 \mathrm{mg}$ TritonX-100/mg protein. The insoluble cell debris was removed by centrifugation at $750 \mathrm{~g}$ for 5 minutes at $4^{\circ} \mathrm{C}$. Solubilized protein $(10-20 \mu \mathrm{g})$ was loaded onto a $10 \%$ glycine/SDSPAGE gel in Laemmli sample buffer. Isolated mitochondria from cells and tissues were solubilized in Laemmli sample buffer prior to loading. Subsequent immunoblotting was done with some of the antibodies described above.

Anti-B17.2L antibody. An antibody against B17.2L was generated by injecting rabbits with a synthetic peptide specific to human B17.2L (CSAPYFGKEEPS), conjugated to keyhole limpet hemocyanin via a reactive sulfhydryl group, and emulsified in Titermax (Pierce Biotechnology Inc.). Testing of the specificity of the B17.2L antisera revealed that affinity purification was not required for immunoblotting, and crude serum was used.

Immunoprecipitation of B17.2L protein from buman heart and human fibroblasts. Anti-B17.2L antibody was affinity purified using the SulfoLink kit (Pierce Biotechnology Inc.). Purified antibody $(70 \mu \mathrm{g})$ was then bound to $20 \mathrm{mg}$ of protein A sepharose $4 \mathrm{~B}$ fastflow (Sigma-Aldrich) overnight at $4{ }^{\circ} \mathrm{C}$. The antibody was cross-linked to the protein A sepharose with $25 \mathrm{mM}$ dimethylpinelimidate in $0.2 \mathrm{M}$ sodium borate, $\mathrm{pH} 9.0$ (30 minutes at room temperature). Cross-linking was terminated with $0.2 \mathrm{M}$ ethanolamine, $\mathrm{pH} 8.0$ ( 3 hours at room temperature). The beads were equilibrated in $20 \mathrm{mM}$ Tris $\mathrm{HCl}, \mathrm{pH} 7.5$, and $1 \mathrm{mM}$ EDTA. Human heart mitochondria $(1 \mathrm{mg})$ were resuspended $(10 \mathrm{mg} / \mathrm{ml}$ protein $)$ in $20 \mathrm{mM}$ Tris- HCl, pH 7.5, and $1 \mathrm{mM}$ EDTA with Complete protease inhibitors (Roche Diagnostics Corp.). An equal volume of $2 \%$ dodecylmaltoside (DDM) in $20 \mathrm{mM}$ Tris $\mathrm{HCl}, \mathrm{pH} 7.5$, and $1 \mathrm{mM}$ EDTA was added, and the mitochondria were solubilized for 30 minutes at $4{ }^{\circ} \mathrm{C}$. Insoluble material was pelleted by centrifugation at $185,000 \mathrm{~g}$. The supernatant was precleared against naked protein A sepharose beads for 1 hour at room temperature, then incubated with $B 17.2 \mathrm{~L}$ protein A sepharose overnight at $4^{\circ} \mathrm{C}$. After washing with PBS, pH 8.0, with $0.05 \%$ DDM, bound protein was eluted from the beads with $0.1 \mathrm{M}$ glycine, $\mathrm{pH} 2.5$, and $0.05 \% \mathrm{DDM}$. Eluent concentration and buffer exchange (PBS, pH 8.0, 0.05\% DDM) was done using a Microcon YM-10 (Millipore). 


\section{Acknowledgments}

We are most grateful to the family for their participation in this study. We thank the many physicians who contributed to the care of this patient, particularly Susan Winter, Neil R.M. Buist, Gamal Boutros, Dorit Bar-Din, Rowena Korobkin, Sakkubai Naidu, Andrea Gropman, and Raymond Coleman; we also thank Douglas Kerr for measurement of pyruvate dehydrogenase activity, Beth Lee for measurement of respiratory chain activities, Gregory Ward for help with software for the genome subtraction, and Mary G. Ripple for interpretation of the neuropathology. We also thank Ariel Abramov for her invaluable contributions to this study. This study was supported by a grant from the
March of Dimes Birth Defects Association to E.A. Shoubridge. E.A. Shoubridge is a senior scientist of the Canadian Institutes of Health Research and an international scholar of the Howard Hughes Medical Institute.

Received for publication June 20, 2005, and accepted in revised form July 26, 2005.

Address correspondence to: Eric A. Shoubridge, Montreal Neurological Institute, 3801 University Street, Montreal, Quebec H3A2B4, Canada. Phone: (514) 398-1997; Fax: (514) 398-1509; E-mail: eric@ericpc.mni.mcgill.ca.
1. Friedrich, T., and Weiss, H. 1997. Modular evolution of the respiratory NADH:ubiquinone oxidoreductase and the origin of its modules. J. Theor. Biol. 187:529-540

2. Abdrakhmanova, A., et al. 2004. Subunit composition of mitochondrial complex I from the yeast Yarrowia lipolytica. Biochim. Biophys. Acta. 1658:148-156.

3. Carroll, J., Fearnley, I.M., Shannon, R.J., Hirst, J., and Walker, J.E. 2003. Analysis of the subunit composition of complex I from bovine heart mitochondria. Mol. Cell. Proteomics. 2:117-126.

4. Gabaldon, T., Rainey, D., and Huynen, M.A. 2005. Tracing the evolution of a large protein complex in the eukaryotes, NADH:ubiquinone oxidoreductase (complex I). J. Mol. Biol. 348:857-870.

5. Grigorieff, N. 1998. Three-dimensional structure of bovine NADH:ubiquinone oxidoreductase (complex I) at $22 \mathrm{~A}$ in ice. J. Mol. Biol. 277:1033-1046.

6. Sazanov, L.A., Peak-Chew, S.Y., Fearnley, I.M., and Walker, J.E. 2000. Resolution of the membrane domain of bovine complex I into subcomplexes: implications for the structural organization of the enzyme. Biochemistry. 39:7229-7235.

7. van den Heuvel, L., et al. 1998. Demonstration of a new pathogenic mutation in human complex I deficiency: a 5-bp duplication in the nuclear gene encoding the $18-\mathrm{kD}$ (AQDQ) subunit. Am.J. Hum. Genet. 62:262-268.

8. Loeffen, J., et al. 1998. The first nuclear-encoded complex I mutation in a patient with Leigh syndrome. Am. J. Hum. Genet. 63:1598-1608.

9. Triepels, R.H., et al. 1999. Leigh syndrome associated with a mutation in the NDUFS7 (PSST) nuclear encoded subunit of complex I. Ann. Neurol. 45:787-790.

10. Schuelke, M., et al. 1999. Mutant NDUFV1 subunit of mitochondrial complex I causes leukodystrophy and myoclonic epilepsy. Nat. Genet. 21:260-261.

11. Loeffen, J., et al. 2001. Mutations in the complex I NDUFS2 gene of patients with cardiomyopathy and encephalomyopathy. Ann. Neurol. 49:195-201.

12. Benit, P., et al. 2001. Large-scale deletion and point mutations of the nuclear ndufv1 and ndufs 1 genes in mitochondrial complex i deficiency. Am. J. Hum. Genet. 68:1344-1352.

13. Benit, P., et al. 2003. Mutant NDUFV2 subunit of mitochondrial complex I causes early onset hypertrophic cardiomyopathy and encephalopathy. Hum. Mutat. 21:582-586.

14. Benit, P., et al. 2004. Mutant NDUFS3 subunit of mitochondrial complex I causes Leigh syndrome. J. Med. Genet. 41:14-17.

15. Kirby, D.M., et al. 2004. NDUFS6 mutations are a novel cause of lethal neonatal mitochondrial complex I deficiency. J. Clin. Invest. 114:837-845. doi:10.1172/JCI200420683.

16. Smeitink, J., Sengers, R., Trijbels, F., and van den Heuvel, L. 2001. Human NADH:ubiquinone oxidoreductase. J. Bioenerg. Biomembr. 33:259-266.
17. Schulte, U. 2001. Biogenesis of respiratory complex I. J. Bioenerg. Biomembr. 33:205-212.

18. Kuffner, R., Rohr, A., Schmiede, A., Krull, C., and Schulte, U. 1998. Involvement of two novel chaperones in the assembly of mitochondrial NADH: ubiquinone oxidoreductase (complex I). J. Mol. Biol. 283:409-417.

19. Janssen, R., Smeitink, J., Smeets, R., and van Den Heuvel, L. 2002. CIA30 complex I assembly factor: a candidate for human complex I deficiency? Hum. Genet. 110:264-270.

20. Ugalde, C., et al. 2004. Human mitochondrial complex I assembles through the combination of evolutionary conserved modules: a framework to interpret complex I deficiencies. Hum. Mol. Genet. 13:2461-2472.

21. Antonicka, H., et al. 2003. Identification and characterization of a common set of complex I assembly intermediates in mitochondria from patients with complex I deficiency. J. Biol. Chem. 278:43081-43088.

22. Scheffler, I.E., Yadava, N., and Potluri, P. 2004. Molecular genetics of complex I-deficient Chinese hamster cell lines. Biochim. Biophys. Acta. 1659:160-171.

23. Schagger, H., et al. 2004. Significance of respirasomes for the assembly/stability of human respiratory chain complex I. J. Biol. Chem. 279:36349-36353.

24. Tsuneoka, M., et al. 2005. A novel Myc-target gene, mimitin, that is involved in cell proliferation of esophageal squamous cell carcinoma. J. Biol. Chem. 280:19977-19985.

25. Hirst, J., Carroll, J., Fearnley, I.M., Shannon, R.J., and Walker, J.E. 2003. The nuclear encoded subunits of complex I from bovine heart mitochondria. Biochim. Biophys. Acta. 1604:135-150.

26. Petruzzella, V., et al. 2001. A nonsense mutation in the NDUFS4 gene encoding the $18 \mathrm{kDa}$ (AQDQ) subunit of complex I abolishes assembly and activity of the complex in a patient with Leigh-like syndrome. Hum. Mol. Genet. 10:529-535.

27. Scacco, S., et al. 2003. Pathological mutations of the human NDUFS4 gene of the $18-\mathrm{kDa}$ (AQDQ) subunit of complex I affect the expression of the protein and the assembly and function of the complex. J. Biol. Chem. 278:44161-44167.

28. Ugalde, C., Janssen, R.J., van den Heuvel, L.P., Smeitink, J.A., and Nijtmans, L.G. 2004. Differences in assembly or stability of complex I and other mitochondrial OXPHOS complexes in inherited complex I deficiency. Hum. Mol. Genet. 13:659-667.

29. Zhu, Z., et al. 1998. SURF1, encoding a factor involved in the biogenesis of cytochrome c oxidase, is mutated in Leigh syndrome. Nat. Genet. 20:337-343.

30. Yao, J., and Shoubridge, E.A. 1999. Expression and functional analysis of SURF1 in Leigh syndrome patients with cytochrome c oxidase deficiency. Hum. Mol. Genet. 8:2541-2549.

31. Tiranti, V., et al. 1999. Characterization of SURF-1 expression and Surf-1p function in normal and disease conditions. Hum. Mol. Genet. 8:2533-2540.

32. Shoubridge, E.A. 2001. Cytochrome c oxidase defi- ciency. Am. J. Med. Genet. 106:46-52.

33. Mootha, V.K., et al. 2003. Identification of a gene causing human cytochrome coxidase deficiency by integrative genomics. Proc. Natl. Acad. Sci. U. S. A. 100:605-610.

34. Antonicka, H., et al. 2003. Mutations in COX10 result in a defect in mitochondrial heme A biosynthesis and account for multiple, early-onset clinical phenotypes associated with isolated COX deficiency. Hum. Mol. Genet. 12:2693-2702.

35. Thorburn, D.R. 2004. Mitochondrial disorders: prevalence, myths and advances. J. Inherit. Metab. Dis. 27:349-362.

36. Leigh, D. 1951. Subacute necrotizing encephalomyelopathy in an infant. J. Neurochem. 14:216-221.

37. van der Knaap, M.S., et al. 1998. Phenotypic variation in leukoencephalopathy with vanishing white matter. Neurology. 51:540-547.

38. van der Knaap, M.S., et al. 2002. Mutations in each of the five subunits of translation initiation factor eIF2B can cause leukoencephalopathy with vanishing white matter. Ann. Neurol. 51:264-270.

39. Sherman, D., Durrens, P., Beyne, E., Nikolski, M., and Souciet, J.L. 2004. Genolevures: comparative genomics and molecular evolution of hemiascomycetous yeasts. Nucleic Acids Res. 32:D315-D318.

40. Guda, C., Guda, P., Fahy, E., and Subramaniam, S. 2004. MITOPRED: a web server for the prediction of mitochondrial proteins. Nucleic Acids Res. 32:W372-W374.

41. Altschul, S.F., et al. 1997. Gapped BLAST and PSI-BLAST: a new generation of protein database search programs. Nucleic Acids Res. 25:3389-3402.

42. Claros, M.G., and Vincens, P. 1996. Computational method to predict mitochondrially imported proteins and their targeting sequences. Eur. J. Biochem. 241:779-786.

43. Miller, A.D., and Buttimore, C. 1986. Redesign of retrovirus packaging cell lines to avoid recombination leading to helper virus production. Mol. Cell. Biol. 6:2895-2902.

44. Lochmuller, H., Johns, T., and Shoubridge, E.A. 1999. Expression of the E6 and E7 genes of human papillomavirus (HPV16) extends the life span of human myoblasts. Exp. Cell Res. 248:186-193.

45. Klement, P., Nijtmans, L.G., Van den Bogert, C., and Houstek, J. 1995. Analysis of oxidative phosphorylation complexes in cultured human fibroblasts and amniocytes by blue-native-electrophoresis using mitoplasts isolated with the help of digitonin. Anal. Biochem. 231:218-224.

46. Schagger, H., and von Jagow, G. 1991. Blue native electrophoresis for isolation of membrane protein complexes in enzymatically active form. Anal. Biochem. 199:223-231.

47. Zerbetto, E., Vergani, L., and Dabbeni-Sala, F. 1997. Quantification of muscle mitochondrial oxidative phosphorylation enzymes via histochemical staining of blue native polyacrylamide gels. Electrophoresis. 18:2059-2064. 